\title{
Study on the Role of Context in Discourse Analysis from the Viewpoint of "Make" in Different Sentence Meanings
}

\author{
Zhou Ling, Tian Chuanmao
}

\author{
School of Foreign Studies, Yangtze University, Hubei, 434023 PRC China \\ *Corresponding Author
}

Received: 14 Oct 2020; Received in revised form: 9 Nov 2020; Accepted: 12 Nov 2020; Available online: 13 Nov 2020

\begin{abstract}
In our real life, we have been performing various verbal communications on different context condition of the discourse analysis. The study of context is derived from the study of language meaning. Then, what is "meaning"? This is a question which scholars have been striving to answer for a long time. In the course of their study, they realized that the explanation of meaning could not be achieved without a full understanding of context. Early in ancient Greek and Rome, scholars began realizing the intimate relationship between language and certain features of situation. We will find out that different words and sentences should carry the same meaning, or vice versa, one word should express different meanings. The key point is whether the selected words or sentences can match the homologous context. If it does, then, sound effects are shown; if not, what we have emerged has no claim to be a successful communication.

Context and text are complementary and mutually dependent. Text, without its specific language environment, is impossible to be accessible to the true meaning of it. This essay will first give a review of the researches on context and discuss the development of scholars' studies of context. The author is going to present the view on context from the point of "make" in different sentences.
\end{abstract}

Keywords-Contexts; discourse analysis; function.

\section{INTRODUCTION}

Language, as the most significant communicative means for human beings, has been employed to perform various functions. Also, its forms and contents are continually enriched with each passing day. With the emergence of new items, especially in the field of economy and technique, new elements and new expressions are added. Therefore, language is a complicated system, where we can find some rules, which is what we call linguistics. However, it may also face a certain subject under different conditions of specific time, occasion, social and historical background. We can find out that even the same sentence, appearing in different context, may be endowed with opposite meanings. The better comprehension and digestion of context appears, the easier for us to catch the deep-seated information the author implies.

\section{LITERATURE REVIEW}

Context is considered as the environment where language exists, functions and develops. It restrains language as well as determines language meaning. It can be classified into two major types, linguistic context and non-linguistic context. The study of language meaning nourishes a long history, actually. Scholars, both abroad and at home, from different fields of philosophy, psychology, linguistics and so on have exerted great effort to the study of context in discourse analysis. They have published multitudinous books or papers on it, and some researches are under the way.

\subsection{Previous Study on Context: Abroad and at Home}

Context is first postulated by anthropologist, which is now evolving into a basic coral concept. In 90s of last century, we formally accept the term "context", but we do not form a systematic acquaintance of context. 
Nida believes that most misunderstandings of expression and discourse are owed to context. We should take verbal factors into account, as well as nonverbal factors. Moreover, in the process of understanding some grammar structure, context is badly significant. Baker considers context as an abstract concept. She thinks that context is a theoretical structure. Specific verbal language can be used to express ideas of specific participants, by which we can express some hints about the close relationship between language and culture. All these characteristics are helpful for readers to taste their own taste of text. That is to say, context decides the sphere of hints which we grasp from the discourse.

As mentioned above, a consensus is achieved that context shares its hold in discourse analysis. Many systemic functional linguists argue that the context of situation from systemic perspectives is targeting at providing a set of discourse analysis to associate the text with context. According to Malinowski, situation refers to the environment where a word, phrase, utterance or even text appears. Firth develops Malinowski's idea and points out that the concept of situation is not to be interpreted in specific terms as a sort of audiovisual record of the surrounding environment but is an abstract representation of environment in terms of certain general categories enjoying relevance with the word, phrase, the utterance or the text.

Then, it is cultural context. It is concerned with the whole social and cultural background against which a word, phrase, utterance or a speech event appears. For instance, while we are watching American soap operas, if the actor or actress says something humorous or witty, the native speakers of audience will laugh in a minute. However, the non-native speakers do not understand the true meaning in joke. Consequently, their facial expressions are mixed. This is mainly because the two kinds of audience do not share knowledge of American customs, traditions, beliefs, values, history or culture.

\subsection{Previous Study on the Discourse Analysis}

Context is the precondition of discourse analysis. That is to say, context exerts many-sided influence on the discourse analysis, in other words, different contexts convey different meanings. Consequently, context and text are complementary and mutually dependent. This article, from the viewpoint of "make" in different discourse meanings, in-depth gives a study on the role of context in discourse analysis.

Here, we first of all make clear the term "discourse". What is a discourse? There are various controversial views on it, and on a similar term "text". In general, people literally interpret "discourse" as a spoken part of language and "text" as a written one.

This essay takes exemptions to prove the article, which shows that the method is not only given for one situation, but various sentence meaning. The way is powerful in identifying the functions of context in the understanding of discourse. It also shows readers the importance of context, and recall more attention should be paid to the understanding of discourse. It also gives some correction to many disapprobative comments and misunderstanding on the context, a new development of pragmatic theory is achieved. Although, it may not be complete, or may also have deficiency and inadequacy, it still has a fine potential for interpretation of utterance and the analysis of discourse.

A consensus is achieved for most linguists that context is a complicated term. Linguists classify the context from different perspectives and for different goals. For example, according to their scopes, context may be classified into broad one and narrow one; according to their content, may it be notional and situational; according to their emotions, may it be emotional and rational; according to their expressing forms, explicit and implicit. Anyhow, context is awfully complicated and complex. Therefore, it is endowed with various functions in discourse analysis.

\subsection{Summary}

Generally speaking, at the beginning of the study of context, it is restricted to the linguistic environment where a linguistic element immediately precedes or follows another. During recent years, the notion of context has been enlarged to various aspects. It may cover the whole text or the whole book. What is more, it even covers the whole physical, social or cultural background. Among the scholars who involved in the study of context, Malinowski and Firth were two important linguists who contributed much to the understanding of the notion of context. The first one suggests that language should be considered as a mode of action, not simply as a matter of stating information. Firth holds the view that the meaning of a discourse does not come from the words which comprise it but from the relation it involved to the context of situation in which the utterance occurs.

There are two known old sayings in English: No context, no text. Each word is a new word when used in a new context. These two sentences tell us that context has played an important role in determining the meaning of word. Considering context's multidisciplinary nature, we may adopt some views of other disciplines when necessary. 


\section{ANALYSIS OF THE MEANING OF 'MAKE' IN VARIOUS STRUCTURES}

Each scholar has their own definition on sentence pattern. For example, Zhao huapu believes that so-called sentence pattern is labeled as a typical sentence structure. We call it typical, because it is abstracted from the meaning and the characteristics of sentence. It is endowed with abstract, typical, and systemic features. What is more, different sentence cherishes different characteristics. Sun, a linguist in China, claims that sentence pattern is the form which should be obeyed and extracted from lots of real sentence in our communication, both oral and written. Therefore, it is typical and universal. All in all, sentence pattern is most likely the same as a discipline which is applied for various kinds of sentences, purified from all kinds of language phenomena. It is no isolated but interrelated with each other. We should know that sentence pattern does not only reflect the discipline constructing the sentence, but also be helpful for the study and utilization of corresponding language learners. Because the language is not isolated, we are able to analyze it from the relationship between word and phrase, clause and text. According to $\mathrm{Xu}$, both in English and Chinese, there are synthetic expression and analytic expression. On one hand, synthetic expression means to use a single word to express a complete and complex concept. While analytic expression means to use a phrase to express a complex concept. Previous research has indicated that Chinese is more reliant on analytic expression than English, because the degree of lexicalization is higher than that in Chinese.

\subsection{Meaning of "make" in SVOC}

In terms of the causative relation, "make + object + complement" is typical in English expressions, besides, “使+宾语+补足语” is common and typical in Chinese. Nevertheless, it can never be said the two structures are totally equivalent. Chinese students of English often have a problem of overusing the sentence pattern like "make + object + complement" inappropriately. It may attribute to the negative transfer of this sentence pattern in Chinese. Few previous studies give exploration to the differences between the two structures. Thus, there is necessity to dig the differences as well as similarities of the two structures. In recent years, corpora have been widely used in applied linguistics, because of its large scale of utilization. Corpora are frequently applied in studies to quantify the possible results and thus make it more convincing. The present study aims to find out the differences and similarities between the two structures on the basis of online platform. The possible results may have important implications for both students' writing and English teaching.
By analyzing the phenomena, it can be found out that there are mainly three kinds of structures when "make" is used as a causative verb, in the following examples, we can see much clearer in the functions of "make" in discourse analysis.

Make + noun (pronoun) + infinitive phrase, -ed participle phrase, propositional phrase, noun phrase, and adjectives or adjective phrase, for instance:

Let us not make ourselves deceived.

It will make you look and feel better.

They make you think of birdies.

It makes him one of the best.

Inverted structure of "make + adjective phrase + noun phrase". In order to keep sentence structures balanced, the long noun phrases are usually removed after complements without formal object "it" in most cases, as the following examples:

\section{And make black my hair.}

In this example, "my hair" is moved after the complement "black" without formal object "it".

Make + it + adjectives + infinitive phrases (clauses). When infinitive phrases or clauses act as objects, it is a need to move them to the positions after the complements with formal object "it" in order to keep sentence structures balanced.

These make it far less likely that they will dive vertically.

The government is also expected to make it easier for foreign investors to take profits out.

Specials make it sound fractionally bigger and deeper.

In the above examples, we are getting to know that the key word "make" is serving as a causative verb. It occurs with words that share the same or similar sematic prosody. Here, "make" serves three different functions in the corresponding discourse analysis.

\subsection{Meaning of "make" in SVO}

As we can see in the above examples, sometimes, people always express what they think directly but in a roundabout way. It is caused by context, which means the communication occurs in a certain occasion. The speakers express indirectly. However, not all indirectness is intentional. We can see that some are launched by linguistic inadequacy. For example, if you want to express something that you think may be harmful to others, you may express it in an indirect way. The use of indirectness in these circumstances can lead the hearer to infer all kinds 
of things about you. In this way, the hearer can get to know what you have implied.

According to Oxford Learner's English-Chinese Dictionary, in English, notional verb refers to the kind of verb which expresses a certain kind of action. It may occur alone, such as the sentence structure as $\mathrm{S}+\mathrm{V}$, I do not know. It may also occur with object, for example, in the structure $\mathrm{S}+\mathrm{V}+\mathrm{O}$, I beat him. Here, the notional verb "beat" is followed by an object him. Besides, the verb can also occur before a complement, for instance, in the sentence,

\section{You make me unhappy.}

The sentence occurs in a way as $\mathrm{S}+\mathrm{V}+\mathrm{O}+\mathrm{C}$. The second word is a verb and it is followed by an object and a complement. These adverbs or prepositions are also sometimes called particles. Most traditional studies on English verbs are conducted from structural approach and focus on their syntactic properties, and in this thesis, we will give an illustration of the function of context in discourse analysis. We are going to extend the discussion from the following examples such as:

\section{I am making a cake.}

I can make a difference.

\section{We can make a new world.}

As we can see in the above examples, "make" serves as a notional verb. And verbs are tended to be classified into two categories. For example, verb can serve as transitive verbs and intransitive verb. The first kind of the verb is the one which cannot collocate with objects directly, and the transitive verb is just the opposite one.

In the first example, we are getting to know that the word "make" is the same as "do". That is the object "cake" determines the meaning of it. As we know, meaning of the word is variable and it is realized at different levels of languages, only the context finally determines which one of the meanings is suitable here. A word may hold many extensive meanings as associative meaning according to Leech. The same discourse can be interpreted into different meanings. Why does it happen? What makes this problem more complex and complicated? The answer is attributed to the context and frequent cases, a specific situation. All of these factors may bestow on a language unit some temporary meaning which is distinct from any other meaning it has under a certain condition. Absolutely, in these cases, the interpretation of a discourse is dependent on the context.

\section{MEANING OF "MAKE" IN CONTEXT}

\subsection{Function as causative verbs}

A causative verb is the one that denotes something to happen. It is often used to describe a situation where the subject "causes" the changes of the action or the state of the object. In linguistics, it is an expression of an agent which causes or forces a patient to perform an action.

In the first sentence structure, "make" means to turn something to another state. It is followed by nouns or pronouns. That is the object of the whole sentence, such as the word you, ourselves, and him. According to Searle, the successful classification of an illocutionary act shows that there are very limited members of basic things we have done with language. We tell people how things are and how things ought to be, we try to get them to do things, we commit ourselves or others to do things, we express feelings and attitudes, besides, we bring about alterations through our utterances.

Then, why do people tend to use indirect verbs instead of more direct causative verbs? We can easily find out that using indirectness is prevailing in our daily language use, which means people do not usually express what they think directly but talk in a roundabout way. This language phenomenon is called the indirectness of language. It is very common in human language communications. Therefore, it can be easily found in any language. What is more, using indirectness in different degrees is illustrated in the following examples:

\section{The wind makes papers scattering.}

There is a condition under which people employ indirectness. Moreover, people choose indirectness out of certain reason when they have a choice between indirectness and directness. This indirect speech is obviously intentional. The examples above belong to this kind. Thus, people have no choice but to use indirect verb to express their feelings and attitudes. This kind of situation is often met in our daily life, certainly. For instance, we will discuss something as a shaped piece of wood fixed across the necks of two animals pulling a cart or plough and so on. Here is an example to start our analysis. The word "make" may be used with other illocutionary forces. For example, the utterance of "the wind makes papers scattering" has an illocutionary force of a warning or a threat. What is more, whenever somebody accused you of stealing money, you will reply that "no, I didn't. I promise you I didn't". It is an emphatic denial. Just like what the beginning of that part says, not all indirectness is intentional, but some is caused by linguistic adequacy, that is to say, for instance, when you do not know the correct word for some object in your own language or a foreign language. On other occasions, we may have to use indirectness because some performance error exists. If you temporarily forget a word, owing to fear, 
nervousness and excitement and so on, you cannot get out. The use of indirectness in these conditions may lead the hearer to infer all kinds of things about you. However, you cannot be called that you have generated any implications. In pragmatics we are more interested only in intentional indirectness.

\subsection{Function as Notional Verbs}

The context may offer explanation for the interpretation of a discourse and it is considered as the explaining function. In order to understand the total exact meaning of a discourse, the hearer should not only acquire the literal meaning of it, but also analyze as well as infer the implied meaning according to the context. The explaining function of context can be shown in the second example. Moreover, in every language, we can fetch many rhetoric uses of language, such as metaphor, tautology, irony and so on. These rhetoric phenomena conceal the real meaning employed by the producer of a discourse behind the literal meaning. To infer the intended meaning and the implication carried by the producer, the receiver has to ask help from the context. This can be seen in the third example.

In the preceding relevance theory, a context is considered as "a psychological construct". Some scholars claim that. "It is these assumptions, absolutely, rather than the actual state of the world, which affects the interpretation of an utterance." It is unquestionable, that psychological construct is characterized by its variability with the progress of communication. In the course of communicating and interacting with each other, the participants all strive to obtain from each new items of information as great a contextual effect as it is, that is to say, context has played a significant role in the process of information conveying. Context is not given but chosen, and the selection of a particular context is determined by the search for relevance information. All in all, the selection of an appropriate set of contextual assumptions is so crucial to the understanding of a discourse and an utterance.

\section{IV.FUNCTION OF CONTEXT IN UNDERSTANDING THE MEANING OF 'MAKE'}

We know that language is a complex phenomenon. In order to understand the true meaning of the context, we have to give a full understanding of the context. Context is the basis of the discourse analysis. Besides, language is a magic and powerful tool used to transmit information, which carries not only syntactic but also semantic information. Language is attached to some additional context. In order to understand the meaning of additional information that the author tries to bring to us, we should give a full acquirement of the context.

In the preceding chapters, we have come to know what context is and the definition of discourse analysis, and what makes the relevance theory a new approach to the study of discourse and human communication. Absolutely, one of the most important aspects is the view on the context, which is vastly different from other related theories. We have also found out that relevance theory attaches more emphasis on the part of hearer instead of speaker. The hearer is who can select and construct an appropriate context in the process of interpreting what the speaker has tried to convey. The notion of context is so important that lots of linguists and scholars, including Halliday, Saussure, and many other scholars, made a large number of researches on it, in order to expound utterances in a most appropriate way. As Brown has proposed that "A variety of approaches in linguistics have illustrated some crucial part of the role that context plays in the interpretation of utterances." Though they hold different views on the contextual factors, they have all studied the notion, both from the social and cultural perspectives.

All communication and interaction among human beings is set in concrete situation or context. Context refers to all specific factors in a communicative situation. It can be divided into two categories. Context plays a crucial role in understanding the special meaning of an utterance. It can effectively guide people to perform much more politely and acceptably in mutual communication and interaction.

As it is, what is context? How can we utilize the context? Context is the specific time, or space, or social and historical background where the communication occurs. Furthermore, it also represents the close relation among the elements in sentence and the article. Language is an important concept in the linguistics. Language is no single. As we all know, the alphabets are combined according to certain disciplines to constitute words, and words make up sentence, and sentence comprise article. Thus, it can be seen that language is a system and its components are related to each other intimately. We cannot analyze the language without its partners, in another words, context. Besides, language is a process of alteration. As time goes by, new elements and expressions emerge, also, the meaning or emotional coloring is changing along with time passing by. That is why human beings are superior to other animals. We have our own verbal language system.

Communicative language learning has probably turned into a prominent methodology and a great guideline for syllabus study in various contexts. With regard to the Chinese context, there is a view holding that 
communicative language study should be adopted to enhance awareness concerning about the concrete time, space, or target. Context is a very complicated branch in linguistics. Its functions should be made most use of in our daily communication and comprehension. In further study and research, we should accumulate the knowledge of context and culture as much as possible, so as to achieve smooth and fluent daily communication and intercultural communication.

\subsection{Identifying the Meaning of a Word}

The importance of context in utterance is self-evident. Only when the language is put in a specific context can the discourse be well understood. Most words require some explanations from their respective linguistic context. Especially the context-bounded words, such as homonyms and polysemy, their meanings dominatingly depend on the situation where it occurs. For example, the adjective word "pretty" has some different meanings as follows:

\section{Pretty late (very late)}

A pretty lady (a beautiful lady)

A pretty piece of music (a sweet piece of music)

A pretty sum of deposit (a very large sum of)

As we can see, without the context, the hearers are not able to grasp the exact meaning of the just word "pretty", because it is a polysemy. However, only if we place the word in a certain collocation, then, it does enjoy a fixed frame, the specific meaning becomes much clearer and regular. The first one phrase, for instance, the word "pretty" modifies person. Thus, we are able to translate it into "beautiful". But the meaning of this word changes, when it is followed by another noun. From the linguistic viewpoint, we've got to know that language is a verbal system which is constituted to convey information according to certain rules. However, we cannot analyze one single, isolated and separated word.

\subsection{Eliminating the Ambiguity}

No matter what the comprehension of one single word or a certain grammar is, we cannot manage it without the help of context. Word is a basic element of language. Sometimes, one word can constitute one sentence. However, in order to correctly grasp the true meaning of the one-single-word sentence, we should turn to the concrete context, such as the specific location of the target sentence, or the collocation.

Context also contributes to identify the ambiguity of the syntactic sentence by some grammatical and structural factors. For example:

(1) Young man and woman ( [young man ]and

\section{[ young woman]; or young [ man and woman])}

\section{(2) I like money than Mary}

\section{I like money more than I like Mary}

\section{I like money more than Mary does}

In the two examples, we can see that each sentence has different meaning in different context, which is called ambiguity by virtue of the structural divisions, logical relationship, the pause and tone and stress. In these situations, context is an essential part in canceling the syntactic ambiguity, as well as making it possible for both the speakers and listeners to correctly understand and communicate with each other. Besides, situation also influences the way of communication. Different occasions determine that we should choose different ways of expression to show our politeness and respect. If you have a notice to all these details, one sentence can help you establish pretty relationship with others, otherwise, what you say may result bad consequence, even make a poor impression on other's minds. A good language learner is the one who can not only study the grammars taking on our communication and writing, but also the language in its correspondent situation. That is why our language system has lived for thousands of years, and why human beings have been studying it all the time. As an old saying goes in China, "every time I study the book, every time I will find something new." That is true of it.

\subsection{Overcoming Cultural Obstacles}

With the great development of international exchanges, people from all walks of life, more or less, take part in the international exchanges. Thus, languages are all under certain context of culture. Communication is no more than language in this degree. It involves translating ideas of different cultural background. Sometimes, culture gap may arouse misunderstanding or block in the communication occurring between two people from different culture. For example, in Chinese, "dog" may be used to express something bad, such as “狗眼看人低”; “丧 家犬”. However, in western society, “dog” refers to something auspicious, for example, if someone is fortunate, we will call him or her "lucky dog". In addition, here is an old saying going as "it rains dogs and cats". From the two opposite altitudes towards "dog", we can see that there exists huge difference among different cultures. We live in one world, but we do not cherish the same way of thinking, customs. Therefore, the depth of the understanding of cultural context determines how well we grasp the true meaning which the author is going to convey to readers. According to Malinowski, a well-known linguist, the language learners should fix great attention on the 
difference between the mother tongue and the target language resulted by culture. The task for a language learner is not only to study the grammars, but also the difference caused by culture, religion, and custom.

At the very beginning, Chinese culture is discussed in a broad sense. The term "culture" is defined as "a transmitted pattern of meaning historically embodied in symbols, which is a system of inherited conceptions, expressed in symbolic forms by means of which people communicate. Culture expresses and develops one's knowledge about the attitude towards life. The social context, also called cultural context, should be considered when discussing about grasping the true meaning of the author. This may result in a conflict for majority of hearers between the native language and target language.

Language is not only a symbol system, but also the carrier of culture. Any kind of language is a composing part of culture. In order to get to know the true meaning of the speaker, we should learn to overcome the cultural gap. Otherwise, it is impossible to continue the communication. Actually, culture permeates every aspect of life. Therefore, cultural context does not only compromise history, philosophy, politics, but also social custom, religion, way of thinking and tradition. Learning a language is to learn what the culture context has reflected. In order to correctly and efficiently master the original information which the author tries to convey to us, one of the most important points is that we should get to know the condition where the dialogue lies in. It is the basis of understanding the discourse. For example, when we are praised by some others, in China, we may say, "no, no I'm not that good" or "no, it is just so-so". Anyhow, Chinese people will deny what they have achieved, even though that is true of them. However, in western society, they will accept all these praises frankly. This is one big difference between eastern society and western society. If we treat a friend from another culture, it may make them feel confusing. In one word, if we want to make a clear understanding of discourse analysis, we should take culture context into account. I will show clearly in the following examples:

A: Do you know the sales manager will be dismissed because of his rude treatment to his clerks?

B: Really? I do not believe it! He does well in his work.

\section{A: If not, I'm a Dutch man.}

In this short essay, the first speaker bets if the manager does not be dismissed, he is a Dutch man. In history, English people have discriminated the Dutch man. Therefore, the phrase "Dutch man" has a little negative sense. As we can see, if we do not know the tradition in
English, it is hard to get a sense of true intention which the author tries to convey to us. Anyhow, context is the basis of discourse analysis. Discourse depends on the context. It is an important part of language learning and research. We should remember that "no context, no text". Besides, every word is a new word in a new context. Only based on different context can we develop our comprehension in discourse analyze. And context, as a complicated branch in linguistics, its function should be made full use of in our daily communication and comprehension. In further study and research, we should accumulate the knowledge of context and cultural as much as possible. Only in this way can we manage to achieve smooth daily communication and intercultural communication.

\section{CONCLUSION}

In our English study, listening is one of the skills which are difficult to master. Listening, as an output skill, is the basic factor for English learner to handle some other skills. It also becomes one significant factor to measure one's level of language study. However, in actual class teaching, many teachers are familiar with the occasions where though the material, grammar and vocabulary are very easy, students cannot make up complete meaning with this scattered information. All that they have got are some fragmentary words or sentences. How does it happen? Actually, in spite of the fact that familiar with the words and grammar, they lack a keen sense of context. Thus, they are stuck in confusion and not able to possess the data they have listened.

Understanding and communication is the way of conveying information. However, nowadays, communication is not only based on the simple oral communication. Sometimes, we cannot understand and grasp the true meaning of the speaker. That is because of the context. In other words, if we do not know the relationship between the text and the related knowledge such as culture background, it perhaps results inappropriateness in certain occasions. Thus, context is the environment of expressing language. It is not only the tool for human beings to know about language, but also equipped with value of study. Our knowledge about context is a process from concrete to abstract, widening and innovating the way of study.

\section{REFERENCES}

[1] Cook, V. Second Language Learning and Language Teaching [M], Beijing: Foreign Language Teaching and Research Press, 2000

[2] George Yule, Pragmatics [M]. Oxford University Press, 
1996 [3] Gavison, Ruth. Legal Protection of Privacy [D]. PhD Dissertation. Oxford: Oxford University, 1975.

[3] Hutchinson, T. and Waters, A. 1987. English for Specific Purposes: A Learning-centered Approach [M]. Cambridge: Cambridge University.

[4] Lyons J. New horizons in linguistics. Harmondsworth: Penguin, 1970

[5] Peccei. J. S. Pragmatics. Foreign Language Teaching \& Research Press \& Routledge, 2000

[6] Sperber, D \&Wilson D Relevance: Communication and Cognition [M] Oxford: Black well publisher.1986

[7] Hatim, B. \& Mason, I. Discourse and the Translator [M]. Shanghai: Shanghai Foreign Language Education Press, 2001

[8] Neubert, Albrecht \& Gregory M. Shreve. Translation as Text. Shanghai: Shanghai Foreign Language Education Press, 2001

[9] Basil Hatim \& Ian Mason. Discourse and the Translator Shanghai: Shanghai Foreign Language Education Press, 2001

[10] Xiehong Bai .COntext and Meaning [J]. Foreign Language Teaching and Research Press, 2000, (4).

[11] Huinan Bao. Cultural Context and Language Translation [M]. Shanghai: Shanghai People's Publishing Press, 1976

[12] Jiwan Bi. Cross-cultural Nonverbal Communication [M]. Foreign Language Teaching and Research Press, 1999.

[13] Shichun Gui, Chunyan Ning. Linguistic Methodology [M]. Foreign Language Teaching and Research Pres, 1997.

[14] Ziran He. Pragmatics and English Learning [M]. Shanghai: Shanghai Foreign Language Education Press, 1996.

[15] Gengshen Hu. Pragmatics of International Communication - From practice to theory $[\mathrm{M}]$. Peking: Tsinghua University Press, 2004.

[16] Zhuanglin Hu. Linguistics a Course Book [M]. Peking University Press, 2001.

[17] Yuxin Jia. The Social Functional Significance of Language, Shanghai: Shanghai Foreign Language Education Press, 1990.

[18] Wangqi Jiang. Pragmatics: Theory and Application [M]. Peking University Press, 2000.

[19] Yunxing Li. Study on the Context of Translation [J]. Shanghai Science and Technology Translation, 1999, (4): 29

[20] Xiaojuan Shi. Cognitive Context and Semantic Understanding. 《Foreign Language Research》 2002

[21] Zhenyu Suo. A Course of Pragmatics [M]. Peking University Press, 2000.

[22] Lina Xu, Context and Interpretation. 《Foreign Language Teaching and Research》

[23] Gangzheng Xizhen. A Collection of Contextual Research Papers. first edition. Beijing Language and Culture University Press, 1992. 9, 10

[24] 兹维金采夫. General Linguistic Compendium. (Pingtie Wu etc. interpret), Peking: Commercial Press, 1981.

[25][25] Yongsheng Zhu. Language-Discourse.Context [M] Tsinghua University Press, 1993 